\title{
Knowledge systems in health in sub-Saharan Africa: results of a questionnaire-based survey
}

\author{
Chris Zielinski, Derege Kebede, Peter Ebongue Mbondji, Issa Sanou, \\ Wenceslas Kouvividila and Paul-Samson Lusamba-Dikassa \\ World Health Organization Regional Office for Africa, PO Box 6, Brazzaville, Republic of Congo \\ Corresponding author: Chris Zielinski. Email: chris@chriszielinski.com
}

\begin{abstract}
Objective: To describe the mechanisms and systems of knowledge acquisition, creation, diffusion, application and improvement of knowledge in two layers of the health system: health policy formulation and the provision of clinical services.

Design: A questionnaire-based survey.

Setting: Health research institutions in 46 countries of the World Health Organization (WHO) African Region.

Participants: Key informants in ministries of health and health care institutions in 39 countries of the Region.

Main outcome measures: Existence of knowledge management infrastructure or components of infrastructure in the countries of the Region.

Results: Central national bodies are always cited as having a major role in policy-setting. Non-national bodies most frequently provide advice and act in monitoring and evaluation, while decentralised entities are strongly involved in dissemination, implementation, and monitoring and evaluation. In general, ministries tend not to have an explicit knowledge management framework and do not map knowledge sources and flows for policy-making. The main sources of evidence are guidelines or recommendations from international or national agencies, although systematic reviews are rarely used. Routine monitoring and evaluation of quality and effectiveness of the process of policy development and its implementation was reported in 19 of the 39 respondent countries. In most respondent countries, electronic medical records were rarely used.

Conclusions: As systematic reviews are rarely utilised as a source of evidence in policy development and few mechanisms exist to assess the performance of the policies, there are significant opportunities for improving policy development.
\end{abstract}

\section{Keywords}

knowledge management, health information systems, health, health research

\section{Introduction}

This paper seeks to provide a description of the knowledge environment of African health systems, based on data from international databases and the results of surveys covering the use of knowledge in two layers of the health system: health policy formulation and the provision of clinical services. The questionnaires covering these aspects were completed by key informants selected according to explicit criteria. In all cases, World Health Organization (WHO) staff at country level acting as focal points for information, evidence and research took the first steps in completing the questionnaires, based on already existing or locally published data.

Since knowledge is an integral component of almost every activity in the health system, it is rarely separated out from the activity and considered as a thing apart. Nevertheless, it is possible to characterise knowledge systems in a simple framework adapted from Landry et al., ${ }^{1}$ involving such elements as acquisition, creation, diffusion, application and evaluation/improvement in a 'knowledge value chain', while recognising that knowledge systems are not linear in nature, as there are feedback loops and dynamic elements of all kinds involved.

Knowledge can be seen in a detailed way as content and as being grouped or managed in a series of systems and mechanisms. In this analysis, we are focusing on the systems rather than the content. This is what we are calling 'knowledge systems in health'.

There are no standards or widely accepted indicators to delineate knowledge systems in the health sector, nor is there an extensive literature on the subject. Our survey offers at best a partial picture of knowledge systems in health in Africa, omitting such areas as the role of knowledge in institutional management and the community, family and individual spheres.

\section{Methods}

The methods used to assess national knowledge systems in health are described in detail elsewhere ${ }^{2}$ but are explained briefly here. 
A survey was carried out on aspects of knowledge systems in health by questionnaire sent to the 46 countries of the WHO African Region (subsequent to the research described in this paper, South Sudan joined the WHO African Region by World Health Assembly Resolution WHA66.21, bringing the total number of countries to 47). The objectives of the survey were to develop evidence for a baseline regarding knowledge creation, acquisition, dissemination, use and improvement. The survey covered the role of knowledge in health policy and in the provision of clinical services. The present analysis and synthesis present the results in these two areas.

It was decided to group countries according to the subregional breakdown adopted by WHO for the 46 countries included in the Region. Since the WHO Regional Office for Africa was envisaging launching national planning of knowledge systems in the regional countries, this aggregation of the data at subregional level complemented the organisational structure, to complement the work of the African Health Observatory (www.aho.afro.who.int).

The 46 countries in the Region are broken down into three subregions as follows:

- Central (11): Angola, Burundi, Cameroon, Central African Republic, Chad, Congo, Democratic Republic of the Congo, Equatorial Guinea, Gabon, Rwanda, Sao Tome and Principe

- South/East (18): Botswana, Comoros, Eritrea, Ethiopia, Kenya, Lesotho, Madagascar, Malawi, Mauritius, Mozambique, Namibia, Seychelles, South Africa, Swaziland, Uganda, United Republic of Tanzania, Zambia, Zimbabwe

- Western (17): Algeria, Benin, Burkina Faso, Cote d'Ivoire, Cabo Verde, Gambia, Ghana, Guinea, Guinea-Bissau, Liberia, Mali, Mauritania, Niger, Nigeria, Senegal, Sierra Leone, Togo

The response rate to the questionnaires was high. In all, 39 countries $(85 \%)$ responded to the health policy questionnaire and 38 countries $(83 \%)$ responded to the clinical services questionnaire.

All countries responded and provided data except Angola, Cameroon and Congo in the Central subregion, Kenya in the South/East subregion, and Niger, Sierra Leone and Togo in the Western subregion, which did not respond.

\section{Results}

\section{Health policy}

Who is involved in knowledge for health policy?. Not surprisingly, central national bodies were always cited as having a major role in policy-setting. Non-national bodies most frequently provided advice and acted in monitoring and evaluation, while decentralised entities were strongly involved in dissemination, implementation, and monitoring and evaluation. Think tanks, task forces and the like played an advisory role in all subregions, as did universities and other academic facilities. Professional associations and unions in the South/East and Western subregions fulfilled advisory and dissemination roles. In the Central subregion they were also involved in policy-setting, while in the Western subregion they both advised and helped set policy, as well as being engaged in implementation, and monitoring and evaluation. Policy networks were not common, although they played an advisory role in policy formulation. Donors and partners played a strong part in all aspects of policy work, from providing advice to evaluation of the results. Non-governmental organisations were similarly involved in all aspects of policy work, particularly in the Central subregion. They were engaged in policy implementation in all subregions. The media's role was considered to be dissemination of policies throughout Africa.

The role of the ministry of health and other central policysetting and implementing bodies. In general, ministries tended not to have an explicit knowledge management framework and, apart from the Western subregion, did not map knowledge sources and flows for policy-making. Regarding national accreditation of medical schools and hospitals and licensing of physicians, this was fairly evenly divided between the ministry of health and a range of other bodies; on occasion both the ministry and another body (such as a physician's council or leading medical institution) were involved. In only 10 of the 39 responding countries $(26 \%)$ did physicians need to undertake continuing medical education to remain licensed. The ministry almost always carried out strategic planning and generally also carried out benchmarking. National guidelines were issued by professional associations in about half of the countries.

\section{Sources and types of evidence}

- Systematic reviews from the Cochrane and Campbell Collaborations. Systematic reviews are key sources of evidence and can have a strong impact in informing policy on health status and service provision. However, these were rarely used in the Region - in only six out of $39(15 \%)$ of the responding countries. Cochrane material was used in universities in Rwanda; by directors in South Africa; in the analysis of the health 
situation in Madagascar; for the 'rational analysis of the evolution of current health policy (health indicators, recurring conditions, current epidemiological picture of the country)' in Benin; and in child health in Ghana

- Guidelines and recommendations from WHO and other multilateral organisations. WHO and other international guidelines were cited by every country $(100 \%)$ as being key sources of knowledge for policy

- Guidelines and recommendations from other international sources and standard-setting bodies. Most countries $(27$, or $69 \%$ ) also used guidelines and recommendations from other international sources and standard-setting bodies

- Guidelines and recommendations from other countries in the Region or regional bodies. Most countries $(24$, or $62 \%)$ also used regional sources

- Guidelines and recommendations from national sources. Almost all countries (35, or $90 \%)$ cited national sources

- Guidelines and recommendations from surveillance data. Again, most countries (30, or $77 \%$ ) used surveillance data

Dissemination, use, and monitoring and evaluation

Dissemination. National health policies were disseminated in various ways. All media were used to some extent, particularly the official gazette of the country $(53 \%)$, radio, TV and speeches (each $51 \%$ ). The Internet was rarely used $(18 \%)$.

Who uses the national health policy? The responses to this question mentioned all stakeholders involved in the health sector.

Formal platforms for social marketing were in use in most countries $(26$, or $67 \%)$.

How is monitoring and evaluation carried out? Routine monitoring and evaluation of quality and effectiveness of the process of policy development and its implementation were reported in most countries in the South/East subregion (10, or $59 \%$ ), but in only three countries $(38 \%)$ in the Central and six countries $(43 \%)$ in the Western subregions.

Methods of collecting feedback on implementation of policy. Mechanisms reported included:

- Quarterly and annual reviews of the ministry of health

- Annual reports on health services

- Annual programme review reports

- Epidemiological bulletins
- Reports of thematic evaluation

- Health information management system reports

- Written feedback and instructions

- Oral on-the-spot feedback

- Progress reports based on National Health Plan indicators

- Publications in the media

- Grassroots consultations

- World Bank supervisory reports

- Meetings of partners in health development

- Stock-taking/evaluation discussed in a workshop in which the intermediate level was brought together with several regional health authorities

- Health policy and associated monitoring and evaluation mechanisms developed along with the National Health Plan

- Supervisory training, periodic internal reviews and health sector reviews with partners

- Quality assurance efforts in the big private hospital groups

- A national monitoring and evaluation committee for the implementation and execution of projects/ programmes

- Surveillance systems

- National health information system

Recent scale-up projects or national campaigns on quality or continuous improvement of health products/ services. Among the activities mentioned to scale-up health services and campaigns on quality or continuous improvement of health products/services, were the following:

- Communicable diseases:

- introduction of community malaria care

- intensive campaigns against malaria, leprosy and Buruli ulcer

- regional project for the prevention and control of sexually transmitted diseases/HIV/AIDS along the migration corridor AbidjanCotonou-Lagos

- World Tuberculosis Day

- campaign to launch or accelerate HIV/AIDS prevention

$\circ$ antiretroviral programme

- Non-communicable diseases:

o anti-tobacco campaigns

- national programme on quality and quality of nursing care

- reinforcement of public and private hospital capacities

- regular epidemiological surveillance and a system of effective response 
- blood donation campaign

- transfusion safety programme

o performance-based financing

- scaling-up of service agreements with non-governmental partners

$\circ$ health cooperatives

- Five Year Socio-Health Programme

o revitalization of post-war healthcare delivery system

- National campaigns for routine immunisation:

- periodic national campaigns of integrated vaccination (distribution of mebendazole) and vitamin A and of impregnated bedsheets

- active research on acute flaccid paralyses in infants with malnutrition, polio

- Campaigns to reduce maternal and neonatal mortality:
$\circ$ family planning
o support programme for reproductive health
- development of plans and standards for delivery and neonatal care
- introduction of Integrated Management of Childhood Illnesses activities
o standards on emergency obstetric care for women in childbirth
- maternal and infant health weeks
o special care for women, especially pregnant women and children aged under five years
o condom use
- Operation Save a Baby

\section{The provision of clinical services}

Knowledge resources in clinical and health services. Regarding the availability of knowledge resources, in most countries electronic medical records were either not used at all, or only used in some hospitals or health centres, and either not at all or only by some physicians in private practice, civil society and the traditional medicine sector. Although electronic medical records can be used to manage health/clinical data and to collect health/clinical information for research and policy development, such use as was observed related principally to managing clinical data.
Teaching hospitals. Teaching hospitals in the Central and Western subregions were reported as generally having few libraries or information centres, books or journals, computers or Internet connections. The situation was markedly better in the South/East subregion, where libraries or information centres were usually available, some information resources and computers were reported, but generally few Internet connections existed.

Health centres. Health centres presented a worse picture than teaching hospitals, with few computers or Internet connections reported anywhere.

Physicians. Physicians in private practice used electronic medical records more often than their public sector counterparts. Overall, 24 countries $(62 \%)$ reported some or frequent use, particularly in the South/East and Western subregions. Nevertheless, few physicians used computers or had Internet connections. However, significant minorities in the South/East and Western subregions reported that many physicians in private practice used computers with Internet connections.

Non-governmental organisations/civil society/ foundations. In the South/East subregion, this group generally made some use of electronic medical records, while the other subregions tended not to use them. Few traditional information resources (books and journals) were reported for this group in the Central and Western subregions, while some were available in most countries of the South/East subregion. Most countries had few computers or Internet connections.

The traditional medicine sector. The traditional medicine sector almost never used medical records (only one country out of 39 reported such use) or other information resources of any kind.

Policy and procedures. The ministry of health was the source of health policy directives received at clinical level and procedural guidance in most countries (79\% and $64 \%$, respectively).

The impact of policy on implementation was evaluated at local level in most countries only in the Central subregion (in five countries, or $71 \%$ ), while less than half did so in the South/East and Western subregions ( $47 \%$ and $36 \%$, respectively).

Education and networking among clinical service providers. In all but one country, meetings and conferences were the main form of communication between clinical care providers in the local 
community and their peers abroad. Telephone and fax came second $(77 \%)$, with other methods ranking below $50 \%$. These other methods included radio communication (two-way VHF radio and radio announcements), informal meetings, leaflets and pamphlets, support missions, letters and TV.

When it came to communications with peers abroad, meetings and conferences again occupied the first place $(87 \%)$, but it is notable that the Internet and email $(86 \%)$ ranked above both telephone/fax $(69 \%)$ and newsletters and specialist journals $(59 \%)$. Other forms of communication with peers abroad mentioned included post, telemedicine, study visits and through WHO.

Local healthcare providers participated in continuing medical education in most $(79 \%)$ countries, and national and local training programmes for health workers also existed in most countries $(79 \%$ and $67 \%$, respectively).

Formal networks for healthcare providers were identified in most countries in the South/East (65\%) and Western (57\%) subregions but in only two countries $(29 \%)$ in the Central subregion.

Knowledge sources and targets. The ministry of health was the chief source of all types of knowledge used by clinical service providers in all of the countries of the Region. Clinical trials and Cochrane Collaboration data were only reported as being used in the Western subregion, and there via the Internet and through journal publications. Drug information was often obtained from the Internet and from other sources outside the country. In the Western subregion, knowledge related to immunisation and disease-control campaigns often came from regional and non-governmental organisation sources, in addition to the ministry of health, while knowledge about healthy lifestyles and behavioural advice also came from outside the country, other ministries and non-governmental organisations.

Printed documents and publications were cited throughout as the main format for all knowledge used by clinical service providers. In addition, in the Central subregion, the Internet was also used for health policy; operational procedures, guidelines and methods; drug information; surveillance; and epidemiological data. In all subregions, oral communications, newspapers, magazines and other informal sources, email and the Internet were cited as the main non-print sources for health alerts and disease outbreak information. Apart from through printed materials, healthy lifestyle and behavioural advice was most frequently transmitted orally (in 27 countries, or $69 \%$ ). Surveillance and epidemiological data were often conveyed through newspapers and informal media in the South/East subregion, and orally and through the Internet and email in the Western subregion.

\section{Discussion}

The very preliminary nature of these data and the experimental nature of the survey make it difficult to draw conclusions that are in any way authoritative. Nevertheless, a few indications can be mentioned.

The present survey covered the knowledge systems at the health policy and clinical practice level, and in these the official national language (invariably Arabic, English, French, Portuguese or Spanish) was the principal vehicle for the delivery of knowledge. Studies covering other layers of the health system will need to address language as an integral part of access to knowledge in the health system.

The fact that systematic reviews are rarely utilised as a source for evidence in policy development appears to be a significant opportunity for improving policy development. This could be addressed through an appropriate training and capacity-building programme. Equally, it could be asked why existing systematic reviews are not considered useful. Is this a case of a knowledge gap or are the reviews not appropriate to the needs of health policy-makers in Africa? Is there a need and scope for an African programme of systematic reviews?

As all the surveyed countries made use of WHO guidelines, it would be interesting to conduct a follow-up study on why they are used so consistently, how they are used, and what impact they have on policy development and practices in Africa.

The processes for monitoring how policies are disseminated and implemented show room for significant improvement. Monitoring and evaluation were rarely mentioned in the questionnaires. Again, this could merit examination for a targeted intervention. Guidelines on health policy could be developed through WHO mechanisms and disseminated through appropriate means, such as adaptation workshops at country level.

Apart from identifying major gaps, a top-level survey of this kind could not focus on the weak health information infrastructure in Africa and its impact on policy, practice and delivery of health services. The lack of Internet access resulting from the low penetration rate clearly has an impact on access to knowledge and its utilisation and needs to be addressed urgently by African and 
external sources. These are well-known gaps in infrastructure in many developing countries but the response to this has been fragmented and not well orchestrated. There could be a role for WHO acting as a knowledge broker with national and international partners.

\section{Conclusions}

The methodology piloted in the surveys, of knowledge in policy-making and clinical services provision, appears to have yielded clear and useful results and potentially offers countries a means to better understand how knowledge in their health sectors is created, acquired, shared, stored, applied and improved. It could be further developed to describe knowledge flows and identify opportunities for improvement in country health sectors. Further work in this area could examine other layers of the system and develop a clear-cut baseline against which to assess the effectiveness of interventions.

\section{Declarations}

Competing interests: None declared

Funding: WHO Regional Office for Africa
Ethical approval: Not required because the survey did not touch on ethical issues requiring individual consent.

Guarantor: $\mathrm{CZ}$

Contributorship: $\mathrm{CZ}$ wrote the paper, DK and PEM reviewed the paper, IS and WK assisted with field work, PSLD reviewed the initial design of the study and provided support and overall leadership.

Acknowledgements: The authors thank WHO Country Office focal persons for information, research and knowledge management for their contribution in coordinating the surveys in countries. Their counterparts in the ministries of health are also acknowledged. These surveys would not have been possible without the active participation of the head of health institutions and their department heads who have given their time and effort to be interviewed. We also acknowledge the contribution of the consultant who prepared the background material for this paper.

Provenance: Not commissioned; peer-reviewed by Sondus Hassounah and Najeeb Al Shorbaji

\section{References}

1. Landry R, Amara N, Pablos-Mendes A, Shademani $\mathrm{R}$ and Gold I. The knowledge-value chain: a conceptual framework for knowledge translation in health. Bull World Health Organ 2006; 84: 597-602.

2. Kebede D, Zielinski C, Mbondji PE, Sanou I, Kouvividila W and Lusamba-Dikassa P-S. Surveying the knowledge landscape in sub-Saharan Africa: methodology. J R Soc Med 2014; 107(suppl. 1): 13-21. 